\title{
Modeling of co-channel interference in bluetooth low energy based on measurement data
}

\author{
Hamid Kavousi Ghafi* (D, Christian Spindelberger and Holger Arthaber
}

\author{
${ }^{*}$ Correspondence: \\ hamid. \\ kavousighafi@joanneum.at \\ EMCE, TU Wien, \\ Gusshausstrasse 25/354, \\ 1040 Wien, Austria
}

\begin{abstract}
The intense use of the $2.4 \mathrm{GHz}$ ISM band by several wireless technologies has resulted in increased co-channel interference between networks operating in this frequency band. The aim of this paper is to investigate modeling techniques of co-channel interference affecting bluetooth low energy devices. The models are derived from recorded interference. Two types of models are introduced: a time domain model utilizing IQ data as reference and a spectrum-based model in which the reference signal is captured in frequency domain by a real-time spectrum analyzer. The recorded interference is also used as a reference to analyze the accuracy of proposed models. The proposed IQ-based model shows a degradation in performance for the environments with dominant bluetooth interfering signals. The frequency-based model not only addresses this problem, but also results in a huge data decimation in recording the interference.
\end{abstract}

Keywords: Interference, Bluetooth low energy

\section{Introduction}

Interference sources, affecting wireless technologies, can generally be classified into two main categories: intelligent and non-intelligent. Intelligent interferences originate from other wireless systems and the non-intelligent interferences are caused by unintentional electromagnetic emissions [1]. The characterization and modeling of both types have been addressed in many publications.

Non-intelligent sources of interference are commonly characterized based on measurement data. For example, authors in [2] used the results of an extensive measurement campaign to empirically model impulsive interferences, caused by electronic devices. In this work, the statistics of peak amplitudes, pulse durations, and interarrival times of interferences are derived by a set of measurements. A similar approach is presented in [3] to model the impulsive interference in digital video broadcasting-terrestrial (DVB-T).

For the intelligent sources, the models are generally developed using analytical methods. For example, the authors of [4] developed a method to evaluate the performance of bluetooth under IEEE 802.11b interference. In this study, propagation conditions, in addition to PHY and MAC-layer parameters, are used to derive the author(s) and the source, provide a link to the Creative Commons licence, and indicate if changes were made. The images or other third party material in this article are included in the article's Creative Commons licence, unless indicated otherwise in a credit line to the material. If material is not included in the article's Creative Commons licence and your intended use is not permitted by statutory regulation or exceeds the permitted use, you will need to obtain permission directly from the copyright holder. To view a copy of this licence, visit http:// creativecommons.org/licenses/by/4.0/. 
probability of packet collisions between two systems. Similar analyzing approaches are employed in $[1,5-9]$ to investigate other interference scenarios. In these studies, it is assumed that the spatial distributions of interfering sources are well defined, which is not realistic in many applications.

The aim of this paper is to create measurement-based models for co-channel intelligent interference, further abbreviated by interference, in BLE systems. We first investigate the possibility of using AWGN signals instead of WLAN and GFSK signals as interference. It is shown that WLAN signals can be replaced by AWGN signals, but this doesn't hold true for GFSK signals. Then, we exploit these results to model interferences by using measured IQ data. To reduce required memory, the model is extended to the case that reference data are measured in spectrum domain. As the measurements are conducted in realistic environments, this approach excludes potential errors exist in analytical models. It is worth noting that spectrum sensing is widely used in the literature to tackle interference effects in wireless technologies. For example, authors in [10] have proposed a method based on optimized FFT and filter banks for sensing the spectrum, and in [11] different efficient energy detection methods for spectrum sensing under non-flat spectral characteristics are proposed and investigated.

The prominent contributions of this paper can be summarized as follows:

- We have modeled the intelligent interferences on BLE systems via a measurement approach.

- The models are extended to frequency domain recordings, which reduce the required data. This provides the possibility of capturing the interference for a longer duration with the same amount of memory.

- To model and generate the interference signals, we use the statistics of the measured data. The modeled interference imitates the impact of the original interference on a BLE receiver with high accuracy.

- We show that signals originated from WLAN sources can be modeled by an AWGN source, which reduces the complexity of the interference model.

The rest of this paper is organized as follows. In Sect. 2, a basic simulation setup is introduced to analyze BLE performance in presence of different interfering signals. In Sect. 3, the performance of a BLE receiver under modulated interfering signals is compared with its performance under the additive white Gaussian noise (AWGN) signal. Based on results from Sect. 3 and a set of measurement data, interference models and their performances are presented in Sect. 4. Section 5 discusses the procedures and possible applications of the models introduced in this work. Finally, Sect. 6 summarizes the results of this paper.

\section{Methods/experimental}

The modeling approaches proposed in this paper are based on a set of measurement data. First, a measurement campaign was made in a university room. It is worth to note that during capture of interference, in addition to typical wireless local area 


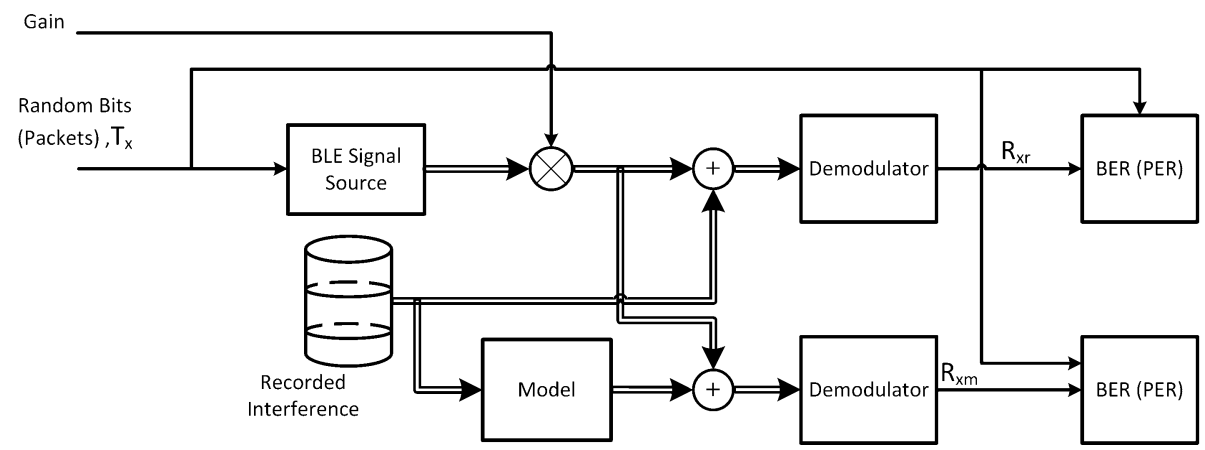

Fig. 1 Simulation setup to analyze BLE performance

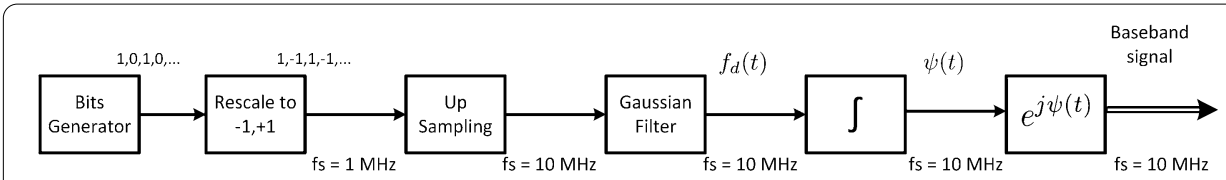

Fig. 2 BLE modulation scheme block diagram

network (WLAN) devices, a few number of bluetooth devices were operating in either advertising or connection mode. The measurement settings are presented in Sect. 2.3. The recorded data are then employed to model the interference.

Figure 1 illustrates a simulation setup to analyze the performance of derived models. For the sake of simplicity, the simulations are conducted in the equivalent baseband. The transmission message (Tx) is modulated and then multiplied by a gain factor to provide the desired signal. Then, the setup compares two conditions. In the first one, the recorded interference signal acts as the interferer. In the second condition, the modeled signal is the interference source. Finally, for both signal paths the same demodulation algorithm is used to retrieve the transmitted message and present the system performance in terms of bit error rate (BER) and/or packet error rate (PER). These parameters are calculated without considering adaptive frequency hopping (AFH) algorithm. This helps to provide a conservative estimate of coexistence between systems [12].

\subsection{BLE signal source}

In order to calculate the BER, a random bit sequence is used as transmit message. For PER calculations, the transmit message consists of BLE link layer packets. Throughout this paper, the PER is derived for short BLE packets with a length of 80 bits. For both message types, the BLE signal-source block employs Gaussian frequency shift keying (GFSK) modulation to derive the baseband signal [13]. Figure 2 shows the block diagram of the BLE modulation algorithm.

The diagram starts with a bits generator which produces a sequence of 0's and 1's with a certain symbol rate, i.e., $1 \mathrm{Msym} / \mathrm{s}$ for basic rate BLE [13]. Then, these bits are mapped to a sequence of -1 's and +1 's. Afterwards, an up-sampling block is used to increase number of samples for each symbol (an over sampling factor of 10 is used in Fig. 2). In the next stage, a Gaussian filter is used to smooth the transition between altering bits. 


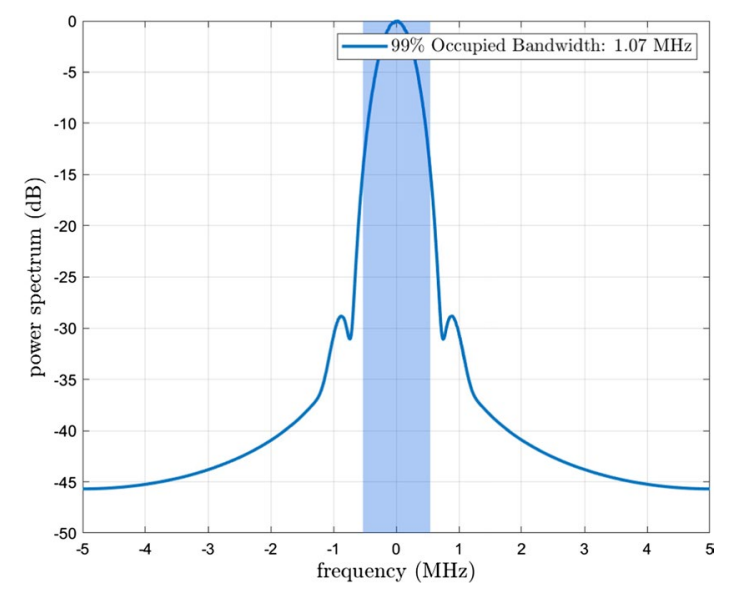

Fig. 3 Frequency spectrum of a BLE signal

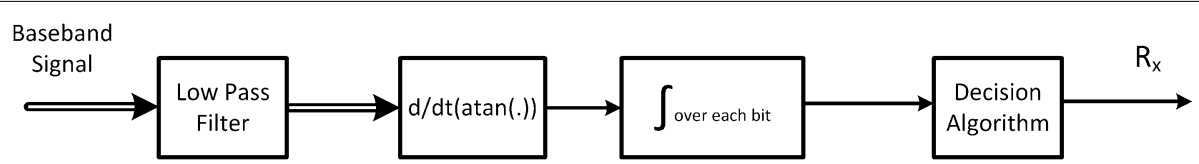

Fig. 4 BLE demodulation scheme block diagram

The output of the Gaussian filter is the instantaneous frequency deviation $f_{\mathrm{d}}(t)$. In the next step, the derived frequency deviation is passed through an integration block to obtain the instantaneous phase deviation $\psi(\mathrm{t})$. Finally, the equivalent baseband signal is calculated using an exponential function. The modulation's output spectrum is illustrated in Fig. 3. As shown in this figure, the $99 \%$ occupied bandwidth is $1.07 \mathrm{MHz}$ and further denoted by OBW.

\subsection{BLE demodulator}

The demodulation is the inverse process of the modulation scheme. In the absence of bit errors, the received message equals the original transmit message. In the literature, several demodulation algorithms are used for BLE systems [14]. The block diagram of a simple algorithm is illustrated in Fig. 4.

First a $2 \mathrm{MHz}$ bandwidth low-pass filter is used to emulate effects of the channel selection filter. Then, the instantaneous frequency deviation is calculated by taking the derivative of the signal phase. In the next processing step, the frequency deviation is integrated over each bit period. Finally, a decision algorithm is used to find the received bit sequence. In this case, the decision algorithm utilizes a simple binary decision threshold. If the integration of frequency deviation over a bit is greater than zero, the decision algorithm maps that bit to ' 1 ,' otherwise it is mapped to ' 0 '.

In the simulation setup, the gain factor is used to realize different signal-to-noise ratios (SNRs) or, equivalently, the bit energy over noise power spectral density $\left(E_{\mathrm{b}} / N_{0}\right)$. By using the $E_{\mathrm{b}} / N_{0}$ parameter, it is implicitly assumed that the interfering signals bandwidth is not smaller than the desired signal bandwidth, which is a reasonable assumption 


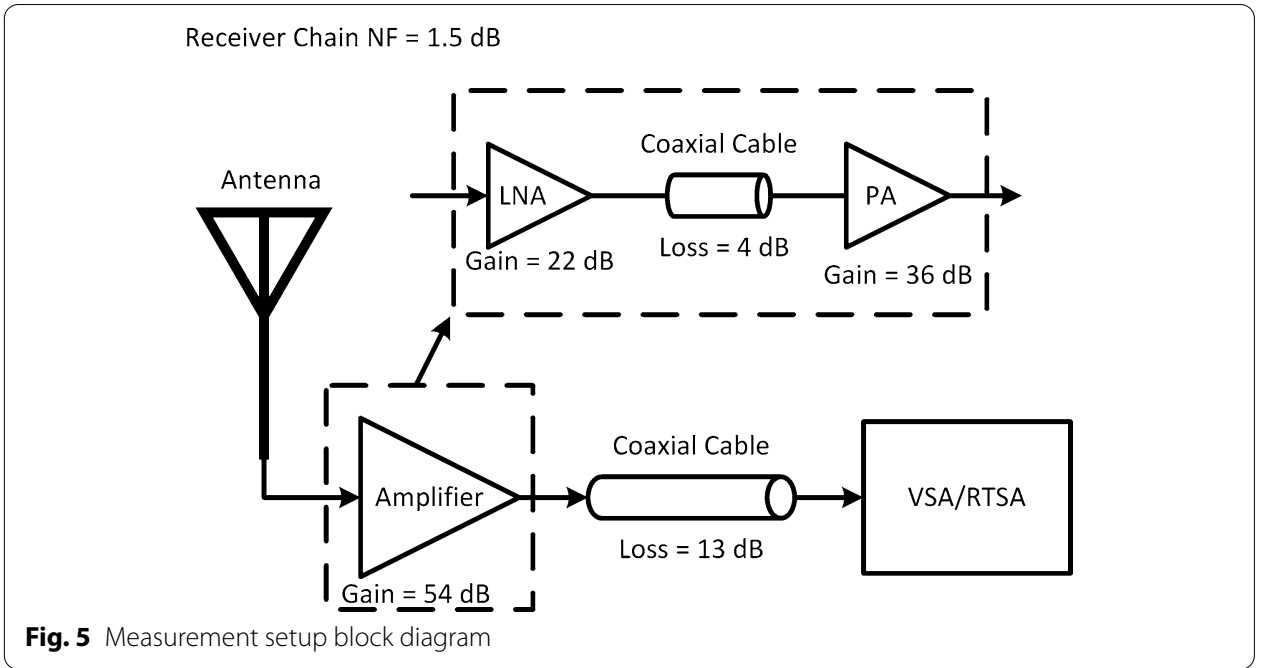

for BLE systems. It is worth to note that in the basic rate BLE, the SNR and $E_{\mathrm{b}} / N_{0}$ are approximately equal:

$$
\mathrm{SNR}=\frac{S}{N}=\frac{E_{\mathrm{b}} \times \mathrm{BR}}{N_{0} \times \mathrm{OBW}} \approx \frac{E_{\mathrm{b}}}{N_{0}},
$$

where $S$ and $N$ are the signal and noise power, $E_{\mathrm{b}}$ is the bit energy, $N_{0}$ is the noise power spectral density, and BR denotes the bit rate.

\subsection{Recorded interference}

Figure 5 shows the functional block diagram of the measurement setup [15]. An omnidirectional antenna is used to capture the signals. As the coaxial cable introduces a highsignal attenuation due to the long cable length, a low noise amplifier followed by a power amplifier was used earlier in the signal path to amplify the signal before sending it to the vector signal analyzer $\left(\mathrm{VSA}^{1}\right)$. The chain is designed so that for the typical interference in the air the PA works in its linear region. However, after recording, this issue is again verified. This design guarantees a low noise figure (NF) and a high gain receiver chain to capture low-power interference events. As a typical BLE receiver has a much higher noise figure than that of the proposed receiver chain, it is reasonable to assume that the setup accurately records all interference events which might influence an imaginary BLE receiver placed at our measurement antenna. Finally, the VSA samples the signal and saves the data in time domain (IQ data). These data are used as reference signal in narrow-band time domain analysis in one hand and, in other hand, is employed to derive the spectrum data for wide-band frequency-based models. To capture the whole ISM band, the data are recorded at the center frequency of $2440 \mathrm{MHz}$ with a span of $80 \mathrm{MHz}$. The IQ data are recorded at sampling rate of $102 \mathrm{MSa} / \mathrm{s}$ because for the used VSA the sampling rate is about 1.28 times of the user span. Due to the limitations in hardware, the maximum time duration of each recording is $2 \mathrm{~s}$. 

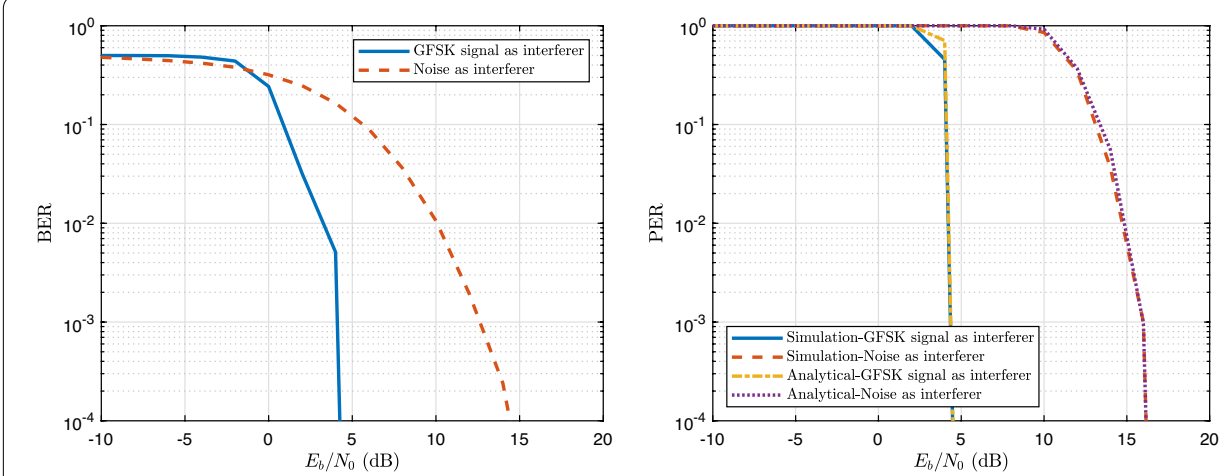

Fig. 6 BLE receiver immunity against GFSK modulated and AWGN interferences. Left: BER, right: PER

\section{AWGN signals for modeling GFSK and WLAN interferences}

The goal of this section is to determine if an AWGN source is capable to emulate various interference signals. In particular, two common interfering signals are studied: the OFDM-modulated signals from IEEE 802.11g-based networks and GFSK-modulated signals from BLE devices. In this paper, the modulated interfering signals are designated by the term "interference" and the term "noise" is used to denote AWGN signals.

\subsection{GFSK signal as interferer}

In the following, the effect of interference among BLE devices is analyzed. Consequently, it is assumed that the interference signal is a GFSK-modulated signal as well as the desired signal. Figure 6(left) shows the BLE receiver performance against noise and GFSK-modulated signal in terms of BERs. The results show that for an AWGN interferer, an $E_{\mathrm{b}} / N_{0}$ greater than $10 \mathrm{~dB}$ is required to achieve BER less than $0.1 \%$. For the GFSK signal, this point is reached at about $4 \mathrm{~dB}$. For lower BER values, the discrepancy between the two curves is even larger. This shows that a BLE interfering signal affects the BLE receiver in a different way compared to noise.

As discussed in Sect. 2, it is possible to calculate the PERs by simulating BLE packets and exposing them to different levels of noise and/or interference. Another approach is to derive the PER analytically, using BER results. For a given packet of length $l$, the packet is considered to be lost if at least one bit error occurs throughout the whole packet.

$$
\mathrm{PER}=1-(1-\mathrm{BER})^{l}
$$

Figure 6(right) illustrates the PER versus $E_{\mathrm{b}} / N_{0}$ for two aforementioned interference scenarios. The figure shows that simulated and analytical results are in a good agreement. This also means that the BER parameter fully describes system performance. Therefore, in the next sections, the BER is used to present BLE performance in different interference scenarios. 


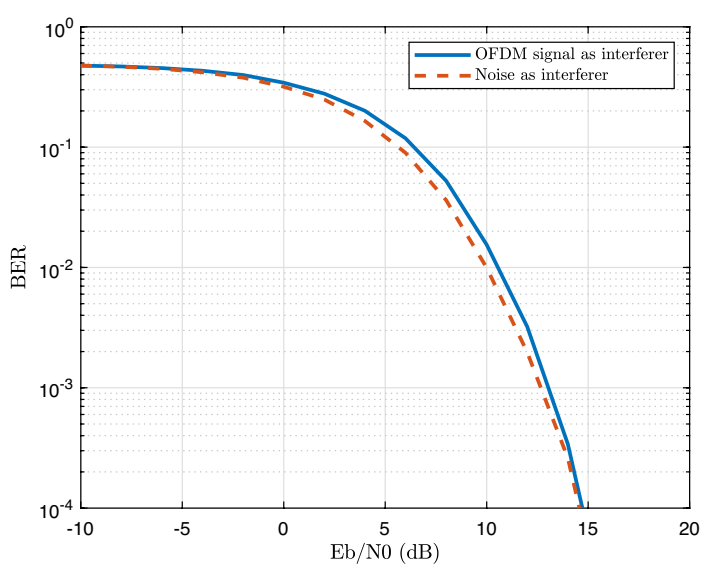

Fig. 7 BLE receiver immunity against OFDM modulated and AWGN interferences

\subsection{WLAN signal as interferer}

In this section, the performance of BLE under the interference of IEEE 802.11g-based networks is evaluated. In order to do that, it is assumed that the interference signal is an OFDM signal with $20 \mathrm{MHz}$ bandwidth. A comparison between the impact of noise and OFDM signals on a BLE receiver is presented in Fig. 7. Because of the large bandwidth and noise-like amplitude distribution of OFDM, the BER curves are approximately equal.

\subsection{Results interpretation}

The simulation results from Sects. 3.1 and 3.2 show that OFDM and noise interfering signals affect BLE systems similarly. Apart from that, the GFSK interfering signals behave differently compared to noise. This makes it difficult to assume that an AWGN source emulates any type of interference in the BLE systems, unlike the claim in [16]. However, these results can be utilized to yield an efficient model for many realistic interference scenarios.

\section{Interference modeling using the reference measurements}

As already indicated in Sect. 2.3, the models proposed in this paper are derived from interference recordings. Two types of interference models are introduced. First are the models that their characteristic parameters are calculated from IQ data. As these models require a huge memory to capture reference signal, the approach is then extended to spectrum-based models.

\subsection{A narrow-band interference model using IQ data}

For the moment, the effects of Bluetooth interfering signals, discussed in Sect. 3.3, are ignored. In other words, it is assumed that a noise source can emulate all interference events in the recorded signal. Given this assumption, a simple modeling approach is to detect the interference events through the recorded data and replace them with AWGN events. In the following, the interference is characterized by its mean power, 

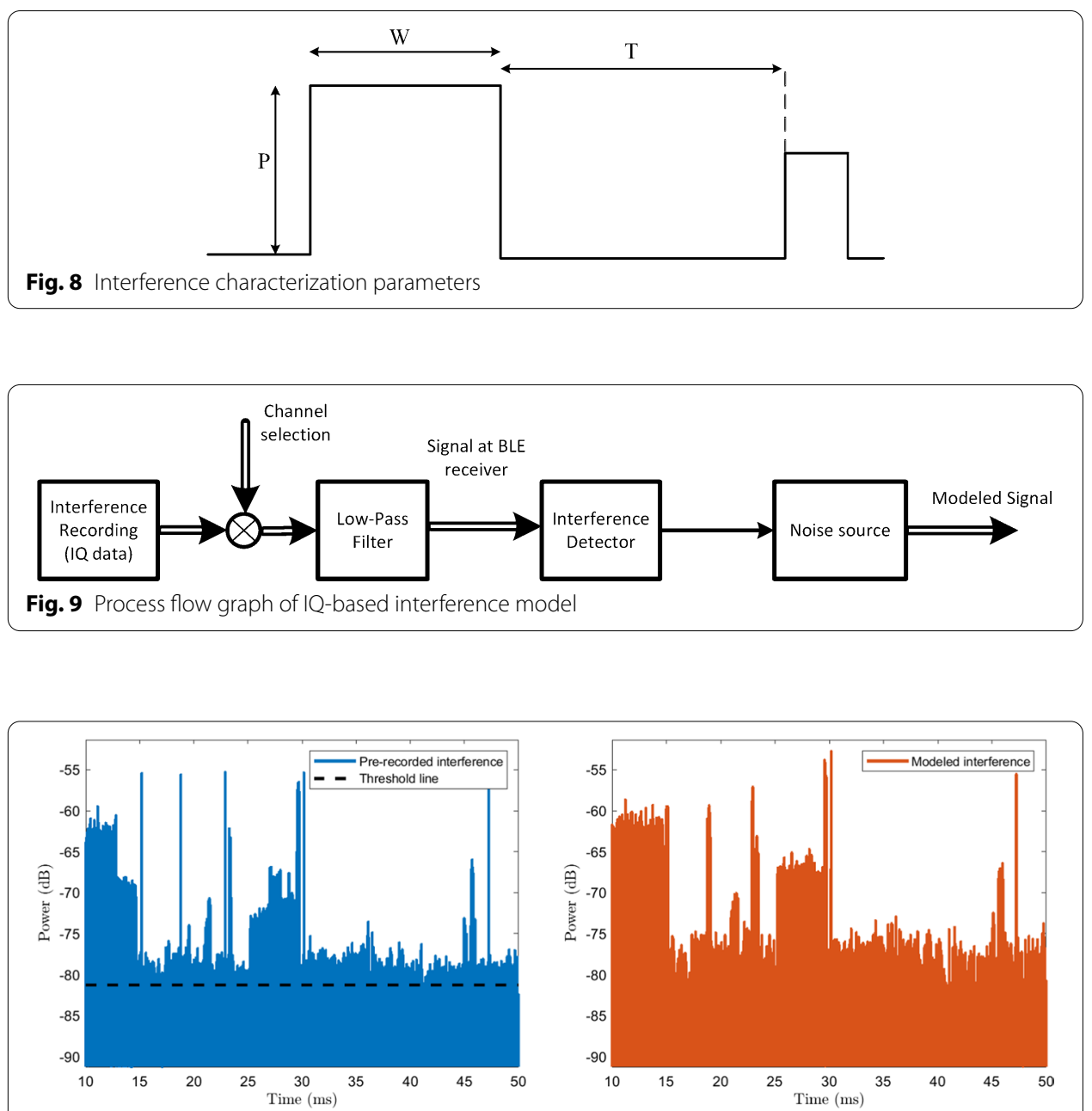

Fig. 10 Envelope power. Left: pre-recorded interference, right: IQ-based modeled interference

time duration (pulse width), and pause to the next event. Throughout this work, these variables are designated by $P, W$, and $T$, respectively (see Fig. 8). The frequency characteristics are ignored because all processing steps are performed for a single channel. Later, in the frequency-based model, the center frequency and bandwidth are added to these characteristic parameters.

The processing flow graph of this modeling approach is depicted in Fig. 9. First, the channel of interest is selected from the wide-band recording by filtering. In the next step, a detection algorithm is employed to extract the interference parameters. Further details about the detection algorithm are discussed later by an example. Lastly, each detected interference is replaced with an AWGN pulse of the same power level and time duration. During pause times, the algorithm utilizes an AWGN signal with a power equal to the instrument noise floor. This assures that the modeled signal represents the recorded signal not only during the interference events but also in pause times when there exist no interference.

In the following example, the processing steps are applied to measure IQ data. The BLE data channel 4, centered at WLAN first channel, is selected for the analysis. This 

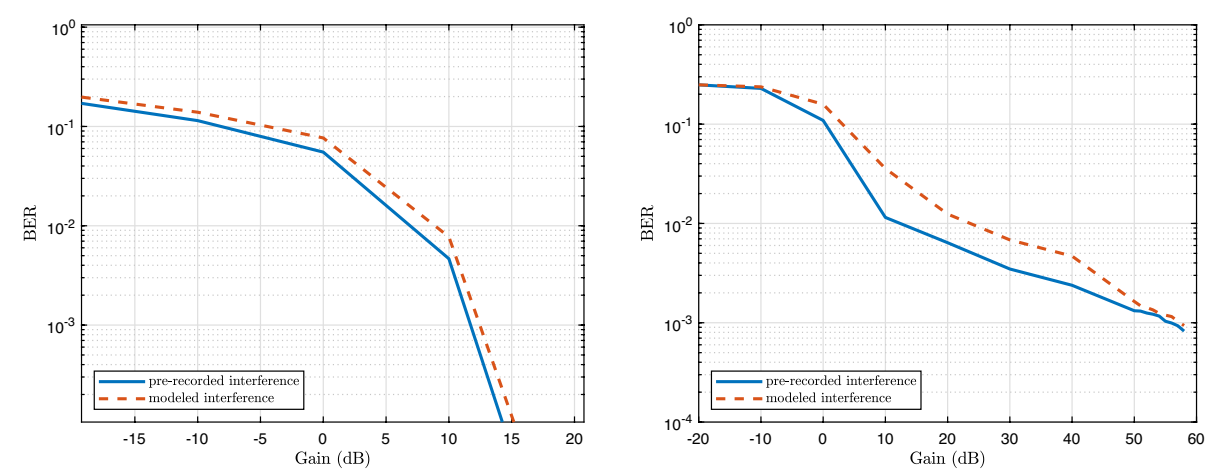

Fig. 11 IQ-based model performance. Left: channel 4, right: channel 39

provides the possibility of testing the modeling approach in a situation with dominant WLAN interference. Later, the model is also examined in a condition that the bluetooth is the main source of interference. Figure 10(left) represents the envelope power of the pre-recorded interference signal, passed through the low pass filter block. To consider a signal as interference, two constraints are applied. First the signal amplitude should be higher than a specified threshold (see Fig. 10(left)). Secondly, it should last for a minimum time period. Setting the constraints properly prevents generating unwanted trigger events, caused by noise. For this example, the threshold is set $6 \mathrm{~dB}$ above the noise floor and the minimum pulse duration is set to $10 \mu \mathrm{s}$.

The envelope power of the modeled interference signal is represented in Fig. 10(right). For power levels below the threshold, the algorithm ignores interferences. In case that several interference signals occur simultaneously, the algorithm models such events as an equivalent signal with the average power level.

Since the interference signal consists of events with different power levels, it is not possible to define a constant signal-to-interference ratio in the simulations. Instead, simulations are performed over a wide range of gain (see Fig. 1). This guarantees the evaluation of all interference events. For example, for high gain values only high-power interference events cause bit errors. As the gain decreases, interference with lower power levels also start to play a role. Figure 11(left) depicts the BER of a BLE receiver versus gain in presence of pre-recorded and modeled signals. The figure demonstrates a good agreement between the results. Doing the simulation for BLE advertising channel 39, centered at $2480 \mathrm{MHz}$, indicates that the model performance is degraded. Figure 11(right) illustrates the results of this simulation. This is because of the fact that the AWGN source fails to accurately model bluetooth interfering signals, as discussed in Sect. 3.3. This problem is addressed in frequency-based model in Sect. 4.2.3.

\subsubsection{Statistical modeling by means of IQ data}

According to what has already been discussed, interference is characterized by $P, W$, and $T$. To statistically model the interference, these variables are assumed to be random. A straightforward approach is to assume that the random variables are uncorrelated, i.e., the CDF of each random variable to be independently calculated. This certainly causes the model performance to degrade. Thus, in this paper, the correlations between random variables are taken into account to improve this technique. 

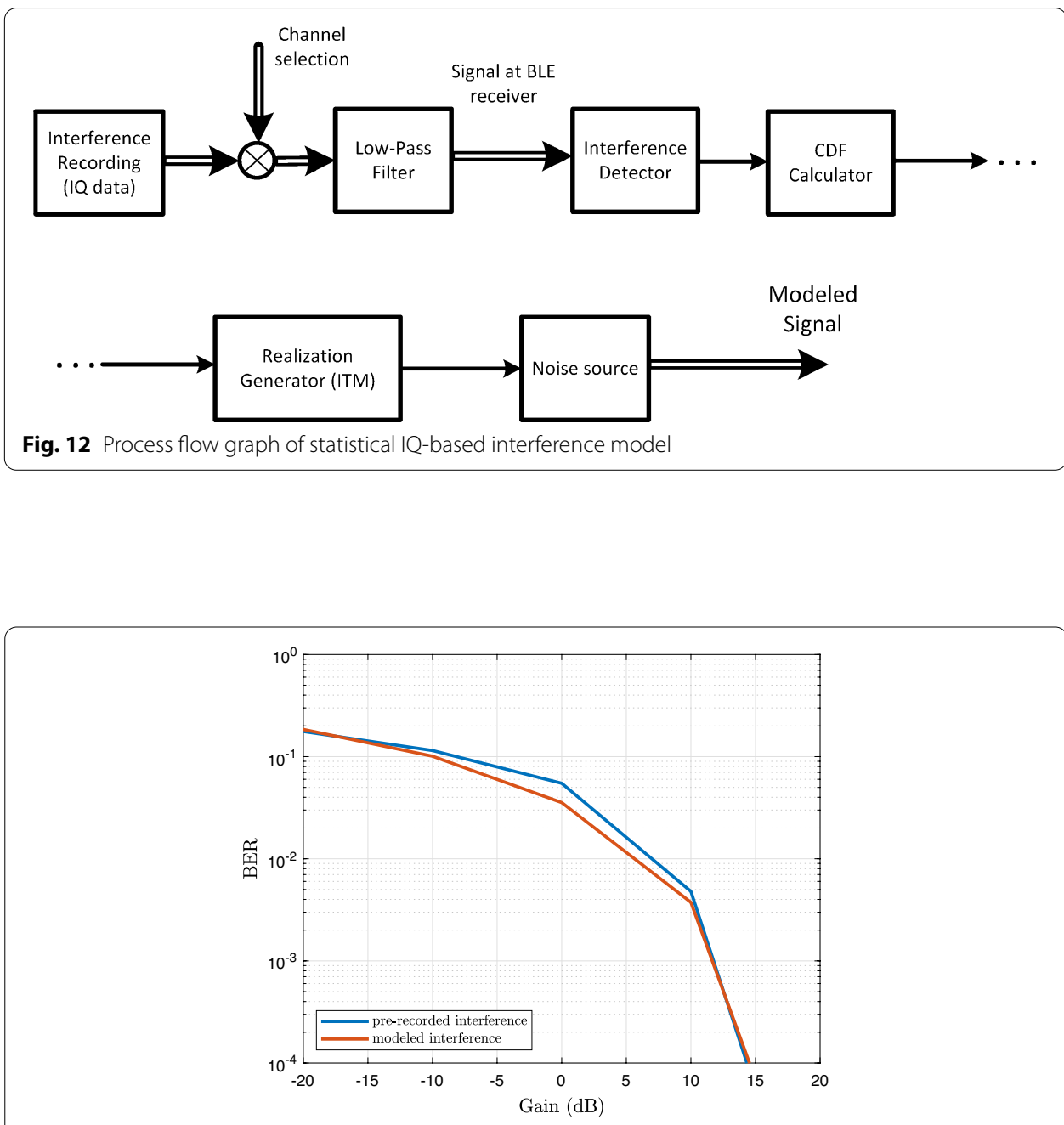

Fig. 13 Statistical IQ-based model performance in channel 4

The statistical modeling process of the interference by means of recorded IQ data is illustrated in Fig. 12. The first three blocks are the same as for the previous section. Nevertheless, the approach to construct an equivalent signal based on the output of the detector block is different. After the detection block, the joint cumulative distribution function (CDF) of random variables is calculated by using a set of detected values. Having the joint CDF of random variables, an arbitrary number of realizations is generated, using the inverse transform method (ITM) [17]. Finally, these realizations and a noise source are used to provide an equivalent baseband signal. This model can be used to generate an interference longer than recorded signal. However, in this situation, change in the statistics of the real-world interference might cause degradation in performance of the model. Figure 13 compares the BER of a BLE receiver in the presence of the pre-recorded and modeled interference at BLE data channel 4. As shown in this figure, this model has a satisfactory level of accuracy in the environments with dominant WLAN interference. 


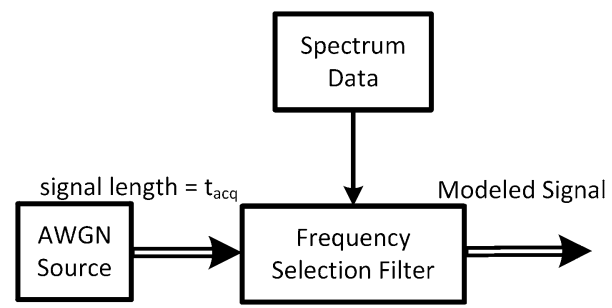

Fig. 14 Flow graph of reconstruction approach based on spectrum data

\subsection{Interference modeling by means of spectrum data}

So far, the models were based on measured IQ data. A direct drawback of such a modeling approach is that a large memory is required to accurately model the interference. In this section, the pre-recorded spectrum data are used to model the interference in order to reduce the required memory. To have a comparable situation with regard to IQbased model, first, the same IQ data are used to generate spectrum data in a similar way as a RTSA does. Then, the frequency-based model is established based on the derived spectrum data.

The RTSA saves the results of successive sweeps (traces). Each trace represents the spectral properties of the signal during an acquisition time period $t_{\text {acq }}[18]$. As a consequence, the problem occurs that short events cannot be precisely measured. In the following, first a simple approach is employed to analyze BLE performance against interference when the spectrum data are available. Then, the impact of acquisition time is investigated in Sect. 4.2.2. Finally, the characterization approach, introduced in Sect. 4.1, is applied to spectrum data to provide a wide-band modeling approach.

\subsubsection{Play back interference using spectrum data}

In this section, a simple algorithm is proposed to find an equivalent baseband signal from spectrum data. This approach can be simply viewed as playing back recorded interference. Afterwards, the impact of acquisition time granularity limitations is addressed.

The processing steps to construct an equivalent signal are presented in Fig. 14. In the rest of this paper, this method is called the reconstruction approach. An AWGN source generates a random noise signal of length $t_{\mathrm{acq}}$. It is again implicitly assumed that each interference event can be modeled via a noise source. Then, this signal is passed through a filter. The filter frequency response is defined by the spectrum data. One can think of this algorithm as a bank of filters that weights the spectrum of noise, corresponding to measured frequency domain data. Considering $X(f)$ as the Fourier transform of the interference signal $x(t)$, the measured spectrum is in the form of absolute value of the $X(f)$, i.e., $|X(f)|$. To model the interference, we use an AWGN signal to make up for the lost phase in the measured data. Hence, by considering $N(f)$ as the Fourier transform of white Gaussian noise $n(t)$ of power 1 , the time domain of the modeled signal can be derived by:

$$
x_{\mathrm{eq}}(t)=\mathfrak{F}^{-1}\{|X(f)| N(f)\},
$$




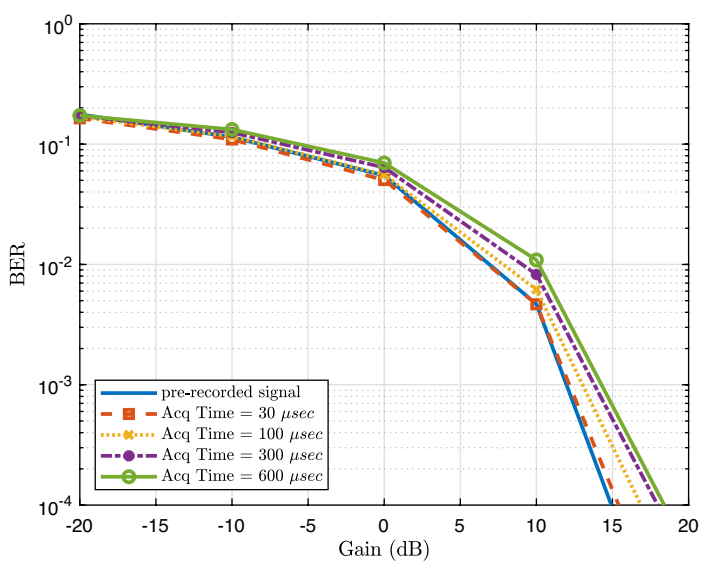

Fig. 15 Acquisition time impact on the model performance

Equation 3 shows how the signal during an acquisition time period is modeled via its spectrum representation and a noise signal. The filter output is considered as an equivalent signal for pre-recorded data during an acquisition time period. For the next trace, the reconstruction approach is similar. Unlike the model proposed in Sect. 4.1, the reconstructed signal represents the interference for the whole ISM band (measurement span), not just a single channel.

\subsubsection{Acquisition time granularity impacts}

The reconstruction approach is potentially limited by the acquisition time granularity of the RTSA. If the acquisition time is equal or less than the shortest interference event, the equivalent signal is expected to reasonably model the interference, regardless of bluetooth interference effects discussed in Sect. 3.3. Nevertheless, for long acquisition time periods, a degradation of the model performance is expected. To assess the impact of acquisition time on model performance, pre-recorded IQ data are used to derive the spectrum data for different acquisition time periods. Afterwards, for each time period, the extracted spectrum data are used to reconstruct an equivalent baseband signal.

For a set of acquisition time periods, the model's performance is depicted in Fig. 15. The simulations are performed for the same data as that of the previous section at BLE channel 4. As shown in this figure, the performance of the model is degraded as the acquisition time increases. This is because of the fact that for long acquisition time periods, there are always some interference events shorter than the acquisition time period. Under such conditions, the algorithm generates longer pulses and consequently a higher BER compared to the actual reference signal. The simulation also indicates that an acquisition time of $30 \mu \mathrm{s}$ is required for an accurate modeling. This value is subject to change depending on the interference scenario. For example, for a lighter occupied environment, a higher acquisition time is required to achieve the same performance.

\subsubsection{A wide-band interference model using spectrum data}

In Sect. 4.1, interference events were detected through recorded IQ data. The events were characterized by power level and timing parameters. The frequency properties 


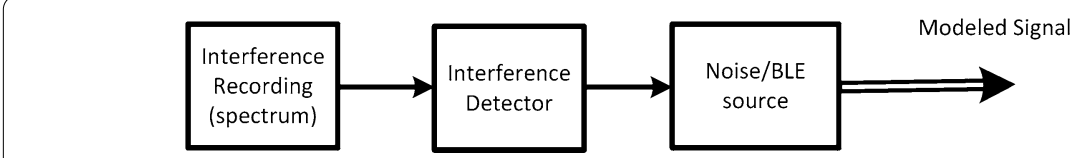

Fig. 16 Process flow graph of spectrum-based interference model

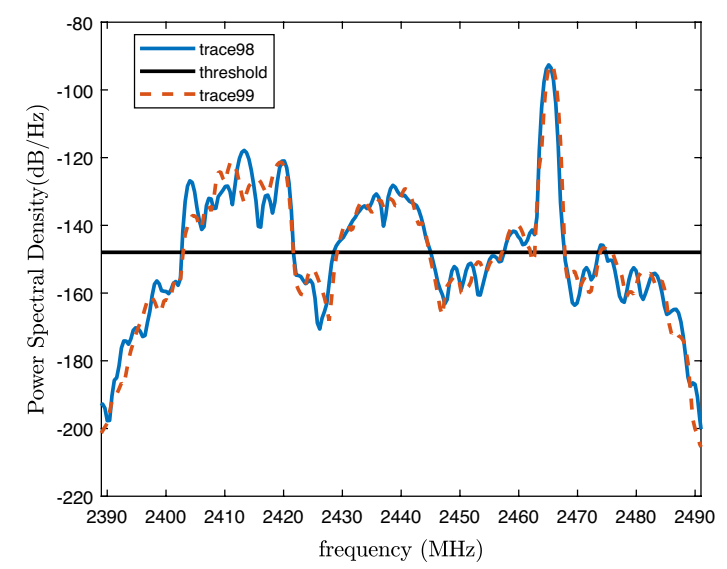

Fig. 17 Power spectral density of recorded interference for two successive traces

were ignored because the analysis was solely done for a single channel. In this section, the interference events are detected through measured spectrum, but this time frequency properties are also included. Each interference is characterized by mean power level $(P)$, timing parameters $(W, T)$, and frequency parameters $\left(\mathrm{BW}, F_{0}\right)$. BW and $F_{0}$ are, respectively, the interference bandwidth and center frequency. Figure 16 shows the processing flow graph of this modeling approach.

First, events through recorded spectrum data are detected. The detected events are divided in three categories based on their BW and $F_{0}$ : bluetooth, WLAN, and other. In the next step, the detected events are used to generate an equivalent-based band signal for interference, similar to the approach used in Sect. 4.1 with two differences. First, the events in bluetooth category are replaced with signals generated by a BLE signal source rather than a noise source. Secondly, as detection is done over the whole band, the output of the model is a wide-band signal which covers the measurement span, not just a single channel.

In the following example, more details about the detection algorithm are presented. Figure 17 shows the spectrum data for two successive traces. Each trace consists of 256 number of frequency bins. The acquisition time is set to $30 \mu$ s. Similar to IQbased model, two constraints are considered to detect interference. First, the $99 \%$ occupied bandwidth of detected event should be greater than a threshold. For this example, this threshold is set to $500 \mathrm{KHz}$. Second, the power spectral density should be higher than a predefined level. This level is set to $6 \mathrm{~dB}$ above the noise floor power spectral density. After detecting events, it is important to correctly associate them through successive traces. In simple words, if an event exists during a few number 

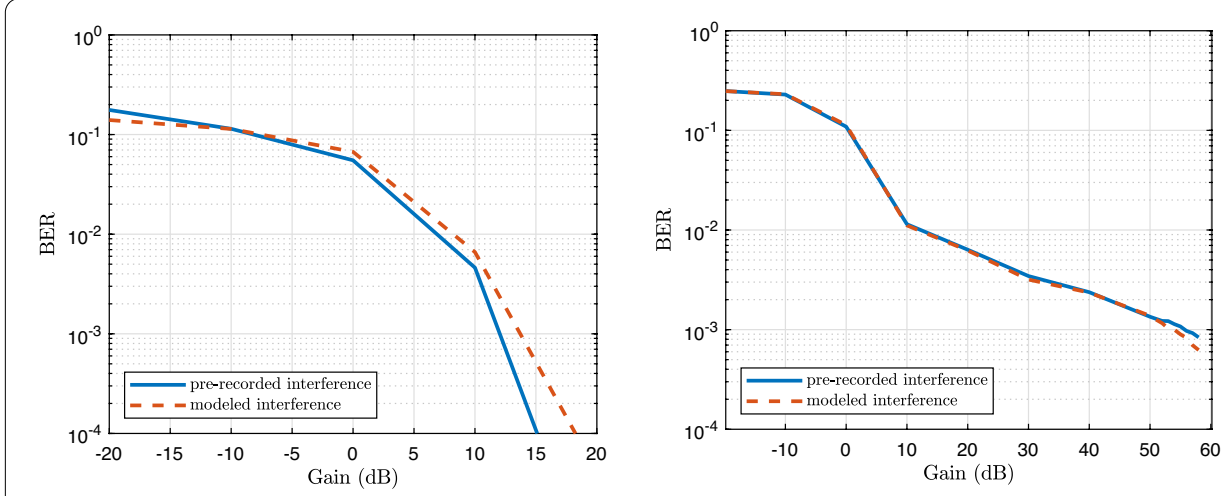

Fig. 18 Spectrum-based model performance. Left: channel 4, right: channel 39

of traces, it should eventually be interpreted as one event. As each trace has $t_{\text {acq }}$ time length, an estimation for the time duration is as follows:

$$
W=N * t_{\mathrm{acq}},
$$

where $N$ is the number of traces in which a certain event is detected. A similar formula is used to estimate pause time. For example, if the start of two events are in a same trace the pause time is estimated as 0 . As a result, the time resolution in calculating timing parameters is $t_{\text {acq. }}$.

In the condition that a bluetooth signal coincides with a WLAN signal, some extra steps are applied to distinguish them. If the bluetooth signal power is much higher than the WLAN signal power, it is expected that $90 \%$ occupied bandwidth is much lower than the $99 \%$ occupied bandwidth. This fact is used to discriminate between two signals. In other side, if the bluetooth signal power is not much higher, according to results from Sect. 3.1, there is no need to detect bluetooth signal. In this situation, the WLAN signal solely models the interference effects.

In the frequency-based model, an important goal is to reduce number of frequency bins. While this decreases the required memory in capturing interference, it leads inaccuracy in detection algorithm. In order to decline number of frequency bins, while keeping the detection algorithm accuracy in a reasonable level, pre-knowledge information about WLAN and Bluetooth signals are employed. For example, the center frequency of detected WLAN and bluetooth events are always shifted to their nearest standard center frequency.

Figure 18 shows the good performance of this modeling approach for both WLAN and bluetooth dominant environments. However, comparing Fig. 18(left) with Fig. 11(left) indicates that the spectrum-based model performs less accurate in BLE channel 4 . This is because of the degradation in detection algorithm for this modeling approach. In other words, due to the high occupancy of this channel, the detection algorithm based on spectrum data is not as accurate as the detection algorithm in IQ-based model. To improve the accuracy of the spectrum-based detection, one solution is to decrease the acquisition time period and/or number of frequency bins. While this improves the accuracy of the detection algorithm, it also results in a higher required memory which contradicts with the aim of this paper. A possible solution, which may be considered 
in future works, is to exploit deep learning algorithms to improve the detection while keeping the data decimation rate (required memory) in a reasonable level. In other side, as shown in Fig. 18(right), this technique solves the problem of inaccuracy in modeling interference originated from bluetooth devices. This implicitly asserts that the detection algorithm, explained above, accurately detects and locates all interference signals for this channel.

\section{Discussion and results}

The immunity against interference is a key factor in the development of BLE technology. Consequently, designers need to carefully assess the performance of the BLE systems against interference. In order to emulate an interference typical to that exist in wireless environments, many researchers have tried to establish standard WLAN and bluetooth networks [19-22]. For example, in [20] a testbed with nine Raspberry Pi3 (RPi3) equally distributed in space is employed to generate bluetooth and WLAN interference. This approach has two main drawbacks. First, it requires substantial efforts to meaningfully set all connection settings. Secondly, the generated signal does not accurately imitate the interference due to the randomness of the real-word interference conditions.

In response to the aforementioned challenges, this paper introduces a new approach to emulate interference. In order to achieve this, goal following steps are undertaken.

- Interference is represented by bursts of events. Events are characterized by a set of parameters that are defined as random variables. These parameters define the amplitude, time, and frequency behavior of the events.

- A measurement campaign is conducted to record interference. The recorded data then are used to derive the statistics of the interference parameters.

- To decimate the required data, the models are extended to the case that recordings are performed in the frequency domain. In this situation, it is possible to capture the interference for a longer duration.

- The derived statistics, in particular joint CDF of random variables, are exploited to generate an interference signal, called also equivalent interference. It is indicated that the modeled interference accurately imitates the impact of the original interference on a BLE receiver.

- It is shown that the events originated from WLAN sources can simply be modeled via an AWGN source. This helps to reduce the complexity of the models in generating equivalent interference.

Another open question is that in which time duration the CDFs of random variables need to be updated. To this end, a massive measurement campaign is required to analyze the variation of statistics for different interference scenarios.

\section{Conclusion}

In this paper, interference in BLE systems has been investigated. In the first step, a measurement campaign in a university room was performed at $2.4 \mathrm{GHz}$ ISM band. The recorded data are used to extract the time and frequency parameters of interference events. This is done both in time and frequency domain. While high-time resolution 
in detecting events is possible in the IQ-based model, the high required memory and processing power restricts it to a narrow-band model. The narrow-band analysis also causes the degradation in the model performance in the environments with dominant bluetooth interfering signals. This can be up to $10 \mathrm{~dB}$ deviation in estimating the impact of real-world interference on a BLE receiver. To address this problem, the modeling approach is extended to a spectrum-based model. Simulations show that it is possible to accurately model interference by capturing highly decimated spectrum data as far as the acquisition time period is lower than a certain value. This value for a typical university room interference scenario is about $30 \mu \mathrm{s}$. The results show that for the spectrumbased model, in the worst case, $3 \mathrm{~dB}$ deviation can be expected in estimating the impact of a real-world interference on BLE receiver. The results of this paper can be utilized by BLE designers to efficiently test and improve their systems for different interference scenarios.

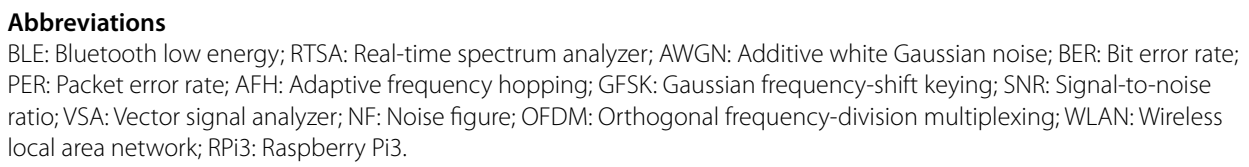
PER: Packet error rate; AFH: Adaptive frequency hopping; GFSK: Gaussian frequency-shift keying; SNR: Signal-to-noise ratio; VSA: Vector signal analyzer; NF: Noise figure; OFDM: Orthogonal frequency-division multiplexing; WLAN: Wireless local area network; RPi3: Raspberry Pi3.

\section{Acknowledgements}

This work was supported by European Regional Development Fund (ERDF) under Project ATCZ175-InterOP. The authors acknowledge the TU Wien Bibliothek for financial support through its Open Access Funding Program. The authors also acknowledge all members of MW laboratory for helps during the research.

\section{Authors' contribution}

HA guided the research and provided the feedback in each step of the work. He also edited the paper before submission. HK performed the simulations and proposed the interference models for BLE systems and also wrote the manuscript. CS participated in measurement campaign and helped to draft the manuscript. All authors read and approved the final manuscript.

\section{Funding}

Funding was provided by European Regional Development Fund (ERDF).

Availability of data and materials

The authors declare that all the data presented in this paper are available.

\section{Declarations}

Competing interests

The authors declare that they have no competing interests.

\section{Authors information}

Not applicable.

Received: 12 February 2021 Accepted: 13 May 2021

Published online: 02 July 2021

\section{References}

1. K. Gulati, Radio frequency interference modeling and mitigation in wireless receivers. PhD thesis (Georgia Tech, 2011)

2. K.L. Blackard, T.S. Rappaport, C.W. Bostian, Measurements and models of radio frequency impulsive noise for indoor wireless communications. IEEE J. Sel. Areas Commun. 11(7), 991-1001 (1993)

3. J. Fernández, Modelling impulsive interference in DVB-T: statistical analysis, test waveforms and receiver performance. EBU technical review (2004)

4. I. Howitt, Bluetooth performance in the presence of 802.11 b WLAN. IEEE Trans. Veh. Technol. 51(6), 1640-1651 (2002)

5. K.P. Subbu, I. Howitt, Empirical study of IEEE 802.15. 4 mutual interference issues, in Proceedings 2007 IEEE SoutheastCon (IEEE, 2007), pp. 191-195

6. K. Gulati, A. Chopra, B.L. Evans, K.R. Tinsley, Statistical modeling of co-channel interference, in GLOBECOM $2009-2009$ IEEE Global Telecommunications Conference (IEEE, 2009), pp. 1-6 
7. D. Dardari, G. Pasolini, Simple and accurate models for error probability evaluation of IEEE802. 11 DS-SS physical interface in the presence of bluetooth interference, in Global Telecommunications Conference, GLOBECOM'02, vol. 1 (IEEE, 2002), pp. 201-206

8. K. Kim, Interference mitigation in wireless communications. PhD thesis (Georgia Institute of Technology, 2005)

9. J. Lansford, A. Stephens, R. Nevo, Wi-Fi (802.11 b) and bluetooth: enabling coexistence. IEEE Netw. 15(5), 20-27 (2001)

10. S. Dikmese, M. Renfors, Optimized FFT and filter bank based spectrum sensing for bluetooth signal, in IEEE Wireless Communications and Networking Conference (WCNC), pp. 792-797

11. S. Dikmese, P.C. Sofotasios, T. Ihalainen, M. Renfors, M. Valkama, Efficient energy detection methods for spectrum sensing under non-flat spectral characteristics. IEEE J. Sel. Areas Commun. 33(5), 755-770 (2015)

12. E. Perahia, S. Li, p802. $11 \mathrm{n}$ coexistence assurance document, doc.: IEEE (2006), pp. 802-11

13. S. Bluetooth, Bluetooth Core Specification v5. O (Bluetooth SIG, San Jose, 2010).

14. R. Schiphorst, F. Hoeksema, K. Slump, Bluetooth demodulation algorithms and their performance, in 2nd Karlsruhe Workshop on Software Radios, vol. 2 (2002), pp. 99-106

15. C. Spindelberger, Interference analysis of WLAN communication. Master's thesis (TU Wien, 2019)

16. J.R. Luque, M.J. Morón, E. Casilari, Analytical and empirical evaluation of the impact of gaussian noise on the modulations employed by bluetooth enhanced data rates. EURASIP J. Wirel. Commun. Netw. 2012(1), 94 (2012)

17. L. Devroye, Sample-based non-uniform random variate generation, in Proceedings of the 18th Conference on Winter Simulation, pp. 260-265 (1986)

18. X. Keysight, S.S. Anayzers, Keysight Technologies (Santa Rosa, 2014)

19. H. Karvonen, K. Mikhaylov, D. Acharya, M.M. Rahman, Performance evaluation of bluetooth low energy technology under interference, in EAl International Conference on Body Area Networks (Springer, 2018), pp. 147-156

20. M. Spörk, C.A. Boano, K. Römer, Improving the timeliness of bluetooth low energy in noisy RF environments, in EWSN, pp. 23-34 (2019)

21. M. Vermeulen, Modeling the coexistence of Co-located BLE and TSCH networks

22. H. Hajizadeh, M. Nabi, R. Tavakoli, K. Goossens, A scalable and fast model for performance analysis of IEEE 802.15. 4 TSCH networks, in IEEE 30th Annual International Symposium on Personal, Indoor and Mobile Radio Communications (PIMRC) (IEEE, 2019), pp. 1-7

\section{Publisher's Note}

Springer Nature remains neutral with regard to jurisdictional claims in published maps and institutional affiliations.

\section{Submit your manuscript to a SpringerOpen ${ }^{\circ}$ journal and benefit from:}

- Convenient online submission

- Rigorous peer review

Open access: articles freely available online

- High visibility within the field

Retaining the copyright to your article

Submit your next manuscript at $\boldsymbol{\Delta}$ springeropen.com 